\title{
Thermal vorticity production in relativistic dissipative fluids *
}

\author{
Yang-guang Yang $^{1}$ and Shi $\mathrm{Pu}^{2}$ \\ ${ }^{1}$ Department of Modern Physics, University of Science and \\ Technology of China, Hefei, Anhui 230026, China \\ ${ }^{2}$ Department of Physics, The University of Tokyo, \\ 7-3-1 Hongo, Bunkyo-ku, Tokyo 113-0033, Japan
}

\begin{abstract}
We have computed the circulation integrations of thermal vorticity with and without charged currents in dissipative fluids. We find that the relativistic Kelvin circulation theorem will be modified by the dissipative effects, therefore, the circulation integrations of thermal vorticity may not be conserved during the fluid evolution.
\end{abstract}

Recently, some novel chiral transport phenomena in relativistic heavy ion collisions have been extensively studied. In a Weyl fermionic system with imbalanced chirality, charged currents can be induced by magnetic or vortical fields, the so-called chiral magnetic or vortical effect $[1,2]$, respectively. In such a system, magnetic and vortical fields also enhance the separation of chirality, which is called chiral separation effect [1, 2]. These phenomena can be described by chiral kinetic equations (CKE), which are obtained by a variety of approaches, such as path integral $[3,4,5]$, Hamiltonian $[6,7]$ and quantum

\footnotetext{
${ }^{*}$ Presented by S. Pu at the Critical Point and Onset of Deconfinement Conference, Wroclaw, Poland, May 30 - June 4, 2016.
} 
kinetic theory via Wigner functions $[8,9,10,11,12]$, see, for example, Ref. [13, 14, 15] for reviews. Similar effects in relativistic quantum system $(2+1)$-dimensional can also be discussed by the quantum kinetic theory [16]. In Refs. [17, 18, 19, 20], in analogy to magnetic fields, the electric fields can also induce a chiral current, the so-called chiral electric separation effect. Similar to normal Hall effects, the chiral Hall effects and the induced density waves are investigated in Ref. [20]. Some new chiral transport effects from the electromagnetic fields up to the second order in the gradient expansion in fluid dynamics have been systemically studied in Refs. [21, 22, 23, 24].

Since an extremely strong magnetic field of the order $\sim 10^{18}-10^{19} G$ is produced in relativistic heavy ion collisions [1, 25, 26, 27], magento-hydrodynamics with chiral transport effects may be an important tool for these phenomena. Some progress has been made along this line. The longitudinal boost-invariant Bjorken flow in $(1+1)$ dimension with a transverse magnetic field with [28] and without magnetization effects [29]. Furthermore, a perturbative solution for Bjorken flow in $(2+1)$-dimension with external magnetic fields has been found in Ref. [30], (also see the recent simulations Ref. [31]).

The vorticial field can lead to the local polarization effect [8]. The vorticity gives the polarization of hadrons at late time of heavy-ion collisions [32, 33]. Several groups estimate the vorticity in different ways, e.g. by the AMPT [34] and HIJING model [35]. In Refs. [36, 37], the thermal vorticity generation in an ideal fluid is discussed. Without charged particles, the circular integration of thermal vorticity is proved to be conserved, which is called relativistic Kelvin circulation theorem. In this note, we will follow Ref. [37] and extend it to a dissipative fluid. We find that the dissipative effects including viscosity and heat conductivity might induce thermal vorticity.

Without charged particles, the energy-momentum conservation reads,

$$
\partial_{\mu} T^{\mu \nu}=0
$$

where $T^{\mu \nu}$ is the energy-momentum tensor and is given by,

$$
T^{\mu \nu}=(\varepsilon+P) u^{\mu} u^{\nu}-P g^{u \nu}+\pi^{\mu \nu},
$$

where $\varepsilon, P$ are energy density and pressure, $u^{\mu}$ is the four-velocity of the fluid, and $\pi^{\mu \nu}$ is the viscous tensor satisfying $u_{\mu} \pi^{\mu \nu}=0$. For simplicity, we only discuss the fluid in the Landau frame, where the heat flux flow $h^{\mu}$ vanishes. Throughout this paper, 
we will use the convention for the metric tensor $g_{\mu \nu}=g^{\mu \nu}=\operatorname{diag}\{+,-,-,-\}$ and the Levi-Civita tensor $\epsilon^{0123}=-\epsilon_{0123}=1$. Then, we have $u^{\mu}$ satisfies $u^{2}=1$, and the orthogonal projector is given by $\Delta^{\mu \nu}=g^{\mu \nu}-u^{\mu} u^{\nu}$. Using the thermodynamic relations $\varepsilon+P=T s$ and $d \varepsilon=T d s$, with $s$ the entropy density and $T$ the temperature, and Eq. (1) in the local rest frame with $u^{\mu}=(1,0)$, a circulation integral along a covariant loop $L(\tau)$ is given by,

$$
\frac{d}{d \tau} \oint_{L(\tau)} T u^{\mu} d x_{\mu}=-\oint_{L(\tau)} \frac{1}{s} \Delta^{\nu \beta} \partial^{\alpha} \pi_{\alpha \beta} d x_{\nu}
$$

where $\tau$ is the proper time defined by $d / d \tau=u^{\mu} \partial_{\mu}$. In the laboratory frame, the fluid velocity becomes $u^{\mu}=\gamma(1, \boldsymbol{v})$ with $\gamma=1 / \sqrt{1-|\boldsymbol{v}|^{2}}$. A similar circulation integration for the thermal vorticity along a loop $L(t)$ is,

$$
\begin{aligned}
& \frac{d}{d t} \int_{S(t)}[\boldsymbol{\nabla} \times(T \gamma \boldsymbol{v})] \cdot d \boldsymbol{S} \\
= & -\oint_{L(t)} \frac{1}{\gamma s}\left[-\gamma^{2} \boldsymbol{v} \partial^{\alpha} \pi_{\alpha 0}+\gamma^{2} \boldsymbol{v}\left(v^{i} \partial^{\alpha} \pi_{\alpha i}\right)\right] \cdot d \boldsymbol{x}+\oint_{L(t)} \frac{1}{\gamma s} \partial^{\alpha} \pi_{\alpha}^{i} d x_{i} .
\end{aligned}
$$

Now let us consider a fluid with charged particles. In presence of electromagnetic fields, the energy-momentum conservation and charge conservation equations read,

$$
\partial_{\mu} T^{\mu \nu}=F^{\nu \lambda} j_{\lambda}, \partial_{\mu} j^{\mu}=0, \partial_{\mu} j_{i}^{\mu}=0
$$

where $j^{\mu}$ is the electric current, $j_{i}^{\mu}$ are other conserved currents and $F^{\mu \nu}=\partial^{\mu} A^{\nu}-\partial^{\nu} A^{\mu}$ is the electromagnetic field strength. For example, if without chiral anomaly, $j_{i}^{\mu}$ can be the chiral current in a Weyl fermionic system. Generally, we can also write,

$$
j_{i}^{\mu}=n_{i} u^{\mu}+\nu_{i}^{\mu}
$$

where $n_{i}$ are the number densities for conserved charges and $\nu_{i}^{\mu}$ are the heat currents. For the electric current, the decomposition is similar, $j^{\mu}=n u^{\mu}+\nu^{\mu}$, with $n$ the electric charge number density, and $\nu^{\mu}$ the heat and electric conducting currents. The thermodynamic relation becomes $\varepsilon+P=T s+\mu n+\sum_{i} \mu_{i} n_{i}$, where $\mu, \mu_{i}$ are the chemical potentials. We follow Ref. [37] to consider the enthalpy vorticity instead of thermal vorticity. We find in the local rest frame, 


$$
\begin{aligned}
\frac{d}{d \tau} \oint_{L(\tau)}\left(f u^{\mu}+A^{\mu}\right) d x_{\mu}= & -\oint_{L(\tau)}\left\{T \partial^{\mu}\left(\frac{s}{n}\right)+\sum_{i} \mu_{i} \partial^{\mu}\left(\frac{n_{i}}{n}\right)\right. \\
& \left.-\frac{1}{n}\left[f u^{\mu}(\partial \cdot \nu)+F^{\mu \lambda} \nu_{\lambda}-\partial_{\nu} \pi^{\nu \mu}\right]\right\} d x_{\mu}
\end{aligned}
$$

where $f=(\varepsilon+P) / n$ is the enthalpy density and in the laboratory frame,

$$
\begin{aligned}
& \frac{d}{d t} \int_{S(t)}[\boldsymbol{B}+\boldsymbol{\nabla} \times(f \gamma \boldsymbol{v})] \cdot d \boldsymbol{S} \\
= & -\int_{S(t)}\left[\boldsymbol{\nabla}\left(\frac{T}{\gamma}\right) \times \boldsymbol{\nabla}\left(\frac{s}{n}\right)+\sum_{i} \boldsymbol{\nabla}\left(\frac{\mu_{i}}{\gamma}\right) \times \boldsymbol{\nabla}\left(\frac{n_{i}}{n}\right)\right. \\
& \left.+\boldsymbol{\nabla} \times \frac{1}{n} f \boldsymbol{v}(\partial \cdot \nu)\right] \cdot d \boldsymbol{S}-\oint_{L(t)} \frac{1}{\gamma n}\left[\partial_{\nu} \pi^{\nu i}-F^{i \lambda} \nu_{\lambda}\right] \cdot d x_{i} .
\end{aligned}
$$

where $\mathbf{B}$ is the magnetic field.

Equations $(3,4,7,8)$ are our main results for the circular integration of thermal vorticity in the local rest and laboratory frame with and without conserved currents.

Now we will focus on a system in the absence of charged particles in the local rest frame. In Eq. (3), without dissipative effects, the circular integration $\oint_{L(\tau)} T u^{\mu} d x_{\mu}$ is conserved, so-called the relativistic Kelvin circulation theorem [36, 37]. It seems that the variation of shear viscous tensor becomes a source to the circular integration of the thermal vorticity. On the other hand, the entropy density in this case is also not conserved. Therefore, we cannot conclude that dissipative effects will violate the relativistic Kelvin circulation theorem. For example, in a longitudinal boost-invariant Bjorken flow in (1+1)-dimension with non-zero shear viscosity, the fluid is homogenous in the transverse plane, i.e. both $T$ and $u^{\mu}$ are independent on the space coordinates $(x, y)$ in the transverse direction. Therefore, any circular integration $\oint_{L(\tau)} T u^{\mu} d x_{\mu}$ along a loop in the transverse plane will vanish during the evolution. However, this result does not hold in a Bjorken flow in $(3+1)$-dimension. So far, we conclude that circulation integration of thermal vorticity may not be conserved due to the dissipative effects.

Then, let us briefly discuss a system in the presence of charged particles in the local rest frame without shear viscous tensor. It is a little surprising that the heat currents $\nu_{i}^{\mu}$ do not appear in the right handed side of Eq. (7), since the electromagnetic fields only couple with electric current $j^{\mu}$.

To end this note, we summarize that we have obtained the dynamical equations for 
the circular integration of thermal vorticity with dissipative effects. In the further con-

siderations, we will learn the generation of thermal vorticity through other approaches, e.g. the kinetic theory and hydrodynamic simulations.

Acknowledgment The authors will thank Qun Wang and Jian-hua Gao for the helpful discussions. S. P. was supported by JSPS post-doctoral fellowship for foreign researchers.

\section{References}

[1] D. E. Kharzeev, L. D. McLerran, and H. J. Warringa, Nucl.Phys. A803, 227 (2008), 0711.0950 .

[2] K. Fukushima, D. E. Kharzeev, and H. J. Warringa, Phys.Rev. D78, 074033 (2008), 0808.3382.

[3] M. Stephanov and Y. Yin, Phys.Rev.Lett. 109, 162001 (2012), 1207.0747.

[4] J.-W. Chen, J.-y. Pang, S. Pu, and Q. Wang, Phys.Rev. D89, 094003 (2014), 1312.2032.

[5] J.-Y. Chen, D. T. Son, M. A. Stephanov, H.-U. Yee, and Y. Yin, Phys.Rev.Lett. 113, 182302 (2014), 1404.5963.

[6] D. T. Son and N. Yamamoto, Phys.Rev.Lett. 109, 181602 (2012), 1203.2697.

[7] D. T. Son and N. Yamamoto, Phys.Rev. D87, 085016 (2013), 1210.8158.

[8] J.-H. Gao, Z.-T. Liang, S. Pu, Q. Wang, and X.-N. Wang, Phys.Rev.Lett. 109, 232301 (2012), 1203.0725.

[9] J.-W. Chen, S. Pu, Q. Wang, and X.-N. Wang, Phys.Rev.Lett. 110, 262301 (2013), 1210.8312.

[10] J.-h. Gao and Q. Wang, Phys. Lett. B749, 542 (2015), 1504.07334.

[11] Y. Hidaka, S. Pu, and D.-L. Yang, Phys. Rev. D95, 091901 (2017), 1612.04630.

[12] J.-h. Gao, S. Pu, and Q. Wang, Phys. Rev. D96, 016002 (2017), 1704.00244.

[13] A. Bzdak, V. Koch, and J. Liao, Lect.Notes Phys. 871, 503 (2013), 1207.7327. 
[14] D. E. Kharzeev, Prog.Part.Nucl.Phys. 75, 133 (2014), 1312.3348.

[15] D. E. Kharzeev, Ann. Rev. Nucl. Part. Sci. 65, 193 (2015), 1501.01336.

[16] J.-W. Chen, J.-H. Gao, J. Liu, S. Pu, and Q. Wang, Phys.Rev. D88, 074003 (2013), 1305.1835.

[17] X.-G. Huang and J. Liao, Phys.Rev.Lett. 110, 232302 (2013), 1303.7192.

[18] S. Pu, S.-Y. Wu, and D.-L. Yang, Phys.Rev. D89, 085024 (2014), 1401.6972.

[19] Y. Jiang, X.-G. Huang, and J. Liao, Phys.Rev. D91, 045001 (2015), 1409.6395.

[20] S. Pu, S.-Y. Wu, and D.-L. Yang, Phys.Rev. D91, 025011 (2015), 1407.3168.

[21] J.-W. Chen, T. Ishii, S. Pu, and N. Yamamoto, Phys. Rev. D93, 125023 (2016), 1603.03620 .

[22] E. V. Gorbar et al., Phys. Rev. D93, 105028 (2016), 1603.03442.

[23] E. V. Gorbar, V. A. Miransky, I. A. Shovkovy, and P. O. Sukhachov, Phys. Rev. B93, 235127 (2016), 1603.06004.

[24] S. Ebihara, K. Fukushima, and S. Pu, Phys. Rev. D96, 016016 (2017), 1705.08611.

[25] W.-T. Deng and X.-G. Huang, Phys.Rev. C85, 044907 (2012), 1201.5108.

[26] V. Roy and S. Pu, Phys. Rev. C92, 064902 (2015), 1508.03761.

[27] H. Li, X.-l. Sheng, and Q. Wang, Phys. Rev. C94, 044903 (2016), 1602.02223.

[28] S. Pu, V. Roy, L. Rezzolla, and D. H. Rischke, Phys. Rev. D93, 074022 (2016), 1602.04953.

[29] V. Roy, S. Pu, L. Rezzolla, and D. Rischke, Phys. Lett. B750, 45 (2015), 1506.06620 .

[30] S. Pu and D.-L. Yang, Phys. Rev. D93, 054042 (2016), 1602.04954.

[31] V. Roy, S. Pu, L. Rezzolla, and D. H. Rischke, (2017), 1706.05326.

[32] L.-G. Pang, H. Petersen, Q. Wang, and X.-N. Wang, Phys. Rev. Lett. 117, 192301 (2016), 1605.04024. 
[33] R.-h. Fang, L.-g. Pang, Q. Wang, and X.-n. Wang, Phys. Rev. C94, 024904 (2016), 1604.04036 .

[34] Y. Jiang, Z.-W. Lin, and J. Liao, Phys. Rev. C94, 044910 (2016), 1602.06580, [Erratum: Phys. Rev.C95,no.4,049904(2017)].

[35] W.-T. Deng and X.-G. Huang, Phys. Rev. C93, 064907 (2016), 1603.06117.

[36] S. M. Mahajan and Z. Yoshida, Phys. Rev. Lett. 105, 095005 (2010).

[37] J.-H. Gao, B. Qi, and S.-Y. Wang, Phys. Rev. D90, 083001 (2014), 1406.1944. 